\title{
Coherent synchrotron emission from electron nanobunches formed in relativistic laser- plasma interactions
}

\author{
B. Dromey ${ }^{1 \star}$, S. Rykovanov ${ }^{2 \dagger}$, M. Yeung ${ }^{1}$, R. Hörlein ${ }^{2,3}$, D. Jung ${ }^{3,4}$, D. C. Gautier ${ }^{4}$, T. Dzelzainis ${ }^{1}$, \\ D. Kiefer ${ }^{2,3}$, S. Palaniyppan ${ }^{4}$, R. Shah ${ }^{4}$, J. Schreiber ${ }^{2,3}$, H. Ruhl ${ }^{2}$, J. C. Fernandez ${ }^{4}$, C. L. S. Lewis ${ }^{1}$, \\ M. Zepf ${ }^{1,5}$ and B. M. Hegelich ${ }^{3,4}$
}

Extreme ultraviolet (XUV) and X-ray harmonic spectra produced by intense laser-solid interactions have, so far, been consistent with Doppler upshifted reflection from collective relativistic plasma oscillations-the relativistically oscillating mirror mechanism ${ }^{1-6}$. Recent theoretical work, however, has identified a new interaction regime in which dense electron nanobunches are formed at the plasma-vacuum boundary resulting in coherent XUV radiation by coherent synchrotron emission $^{7,8}$ (CSE). Our experiments enable the isolation of CSE from competing processes, demonstrating that electron nanobunch formation does indeed occur. We observe spectra with the characteristic spectral signature of CSE-a slow decay of intensity, $I$, with high-harmonic order, $n$, as $I(n) \propto n^{-\mathbf{1 . 6 2}}$ before a rapid efficiency rollover. Particle-in-cell code simulations reveal how dense nanobunches of electrons are periodically formed and accelerated during normal-incidence interactions with ultrathin foils and result in CSE in the transmitted direction. This observation of CSE presents a route to high-energy XUV pulses ${ }^{7,8}$ and offers a new window on understanding ultrafast energy coupling during intense laser-solid density interactions.

Relativistic laser plasmas have been shown to support the generation of bright beams of XUV radiation with attosecond duration $^{9}$ and high spatial quality ${ }^{10}$ in reflection. Experimental observations $5^{5,10,11}$ in the spectral region above the maximum plasma frequency of the interaction ${ }^{12}\left(\omega_{\text {pmax }}\right)$ are consistent with a relativistic Doppler effect provided by a highly reflective critical density plasma surface collectively oscillating in the intense field of the incident laser puls $\mathrm{e}^{1-4}$. This periodic upshifting of the driving radiation into the specularly reflected direction results in harmonic spectra consistent with the relativistically oscillating mirror (ROM) mechanism characterized by $I(n)_{\mathrm{ROM}} \propto n^{-8 / 3}$ scaling $^{4,5}$, where $n$ is the harmonic order. It should be noted that there is another mechanism that can dominate for frequencies below $\omega_{\text {pmax }}$ of the target-coherent wake emission. Here we exclusively discuss harmonic frequencies above $\omega_{\text {pmax }}$ for our interaction densities.

Studies into how laser energy couples to relativistic plasmas, however, have revealed a fundamentally different mechanism for coherent XUV/X-ray generation. Simulations show that dense nanobunches of electrons can be periodically formed and accelerated during each laser cycle at the vacuum-plasma interface for oblique incidence interactions ${ }^{13}$. Conditions have been identified (namely ultrashort plasma-density scale lengths) for which such nanobunches can emit bursts of CSE in specular reflection for few-cycle $(<5)$ interactions ${ }^{7}$. CSE from relativistic laser plasma interactions is predicted to be characterized by a slowly varying, synchrotron-like harmonic spectrum scaling as $I(n)_{\mathrm{CSE}} \propto$ $n^{-4 / 3}$ to $n^{-6 / 5}$ up to a rollover frequency $\omega_{\mathrm{rs}}$ that is intrinsically linked to $\delta$, the spatial extent, and $\gamma_{\max }$ the maximum relativistic Lorentz factor, of the nanobunch. Above $\omega_{\text {rs }}$ the spectrum exhibits a very rapid exponential rollover ${ }^{7,8}$.

In this regime coherent XUV/X-ray emission can no longer be described by a relativistic Doppler upshift from a smooth critical-density-plasma surface oscillating in the laser field. Instead CSE relies on the formation and rapid acceleration of dense electron nanobunches at the relativistic plasma surface by the driving laser field for bright XUV/X-ray generation ${ }^{14}$.

Unfortunately, isolating the CSE mechanism in specular reflection during oblique incidence interactions is not straightforward from an experimental perspective. The requirement of high interaction intensity $\left(\gg 10^{18} \mathrm{~W} \mathrm{~cm}^{-2}\right)$ at present typically implies the use of many-cycle laser pulses $(\gg 5)$, which in turn results in evolving plasma parameters, in particular the plasma density profile becoming comparable to $\lambda_{\text {laser }}$, the laser wavelength. This evolution prevents the specific conditions ${ }^{7,8}$ required for CSE generation from being maintained (or even attained) over the entire pulse duration. On the contrary, the evolution of the plasma towards relatively longer density scale lengths favours the ROM mechanism to dominate $\mathrm{e}^{14-17}$ over CSE during the interaction. Consequently, characteristic CSE spectra in reflection from relativistic laser plasma interactions have not been observed so far.

To overcome this problem we investigate an interaction geometry where the relativistic Doppler effect cannot play a role-normal incidence interactions with ultrathin foils where coherent XUV radiation is studied in transmission (Fig. 1a). In the transmitted direction it is not possible to observe emission due to ROM Doppler upshifting because this can occur only if there is a reflective surface moving towards the incident radiation. Previous work on the mechanisms of harmonic generation in transmission from foils has been predominantly examined for oblique incidence interactions ${ }^{14,18,19}$.

\footnotetext{
1Department of Physics and Astronomy, Centre for Plasma Physics, Queens University Belfast, BT7 1NN, UK, ${ }^{2}$ Department für Physik, Ludwig-Maximilians-Universität, Am Coulombwall 1, D-85748 Garching, Germany, ${ }^{3}$ Max-Planck-Institut für Quantenoptik, Hans-Kopfermann-Str. 1, D-85748 Garching, Germany, ${ }^{4}$ Los Alamos National Laboratory, Los Alamos, New Mexico 87545, USA, ${ }^{5}$ Helmholtz Institute Jena, 07743 Jena, Germany. †Present address: Lawrence Berkeley National Laboratory, Berkeley, California 94720, USA. *e-mail: b.dromey@qub.ac.uk.
} 


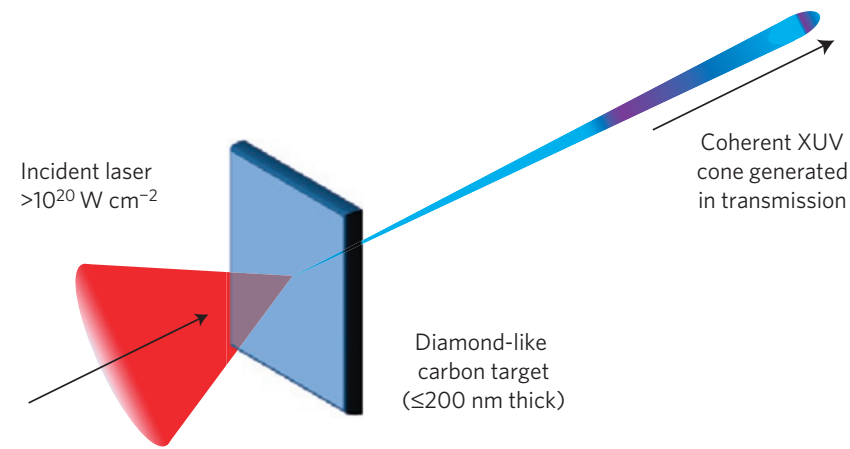

b

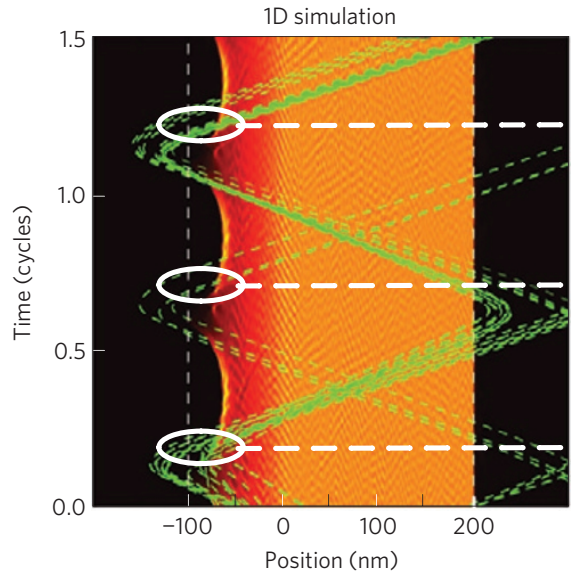

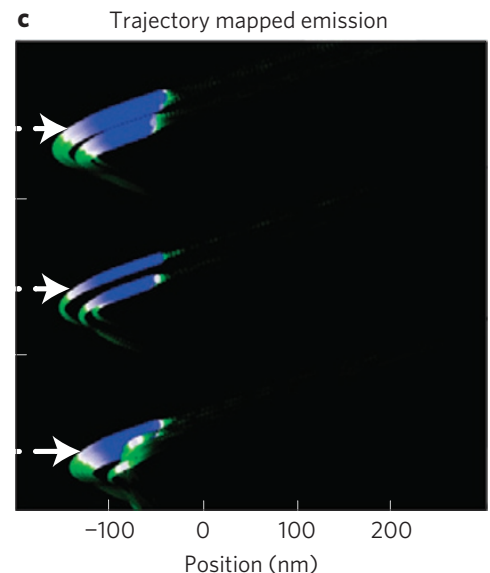

d

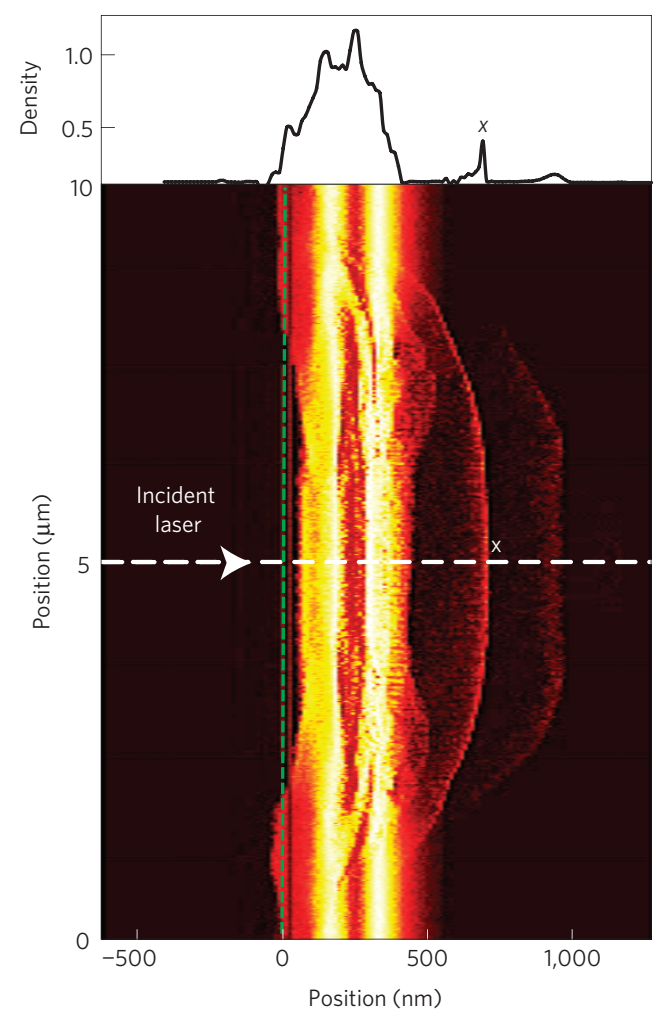

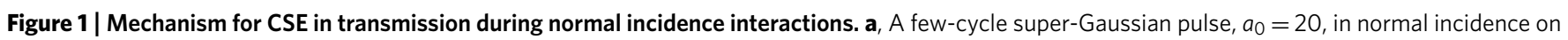
a 200-nm-thick solid-density $\left(800 N_{c}\right)$ foil with a $100 \mathrm{~nm}$ linear pre-plasma ramp to vacuum is studied using the PICWIG $1 D / 3-P$ PIC code 20 . See Methods for the definition of $a_{0}$ and $N_{c}$. $\mathbf{b}$, The trajectories of those quasi-electrons that gained the longitudinal momentum $p_{x}>5$ during 1.5 laser cycles. c, The intensity of emission with respect to position and time from the same simulation parameters extracted from the nanobunch trajectories (blue

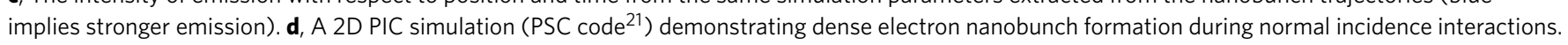
This simulation is performed for a $50 \mathrm{fs}$ pulse with $a_{0}=20$, target thickness $150 \mathrm{~nm}$ with a $100 \mathrm{~nm}$ linear ramp to vacuum on the front and rear surfaces, and density $40 N_{c}$. The density profile is measured along the white dashed line (d) and the bunch width $\delta$ is measured at $x$. The CSE mechanism is discussed in greater detail in the Supplementary Information.

The fundamental mechanism for coherent XUV/X-ray generation in this geometry is studied using particle-in-cell (PIC) $\operatorname{codes}^{20,21}$ and is detailed in Fig. 1b-d. Dense nanobunches of electrons are formed at the front surface of the target and accelerated into the transmitted direction owing to the relativistic ponderomotive force of the laser (Fig. 1b). This observation is in excellent agreement with previous work ${ }^{22}$. These nanobunches undergo rapid elliptical trajectories across the plasma-vacuum boundary before being emitted through the rear of the foil in the transmitted laser direction. Examining the temporal evolution of radiation emitted from a given trajectory reveals an intense, ultrafast $(<0.1$ laser cycle duration) XUV pulse emitted in the transmitted direction just as the bunch reverses its direction towards the target. This is shown by the blue regions in the XUV emission intensity mapped onto the bunch trajectories (Fig. 1c). The nanobunches are observed to have a spatial extent at the resolution limit of the simulation of $\delta \sim 10 \mathrm{~nm}$ and a density $>0.4 N_{\max }$ (where $N_{\max }$ is the maximum target density, Fig. 1d).

Figure 2 shows the spectrum and corresponding temporal structure generated in transmission during the rising edge of a $500 \mathrm{fs}$ laser pulse in normal incidence on an ultrathin foil from PIC simulations. The spectrum is characterized by a lowfrequency cutoff (corresponding to $\omega_{\text {pmax }}$ of the parent foil) and an efficiency scaling slope closely following the $n^{-4 / 3}$ predicted for CSE in specular reflection ${ }^{7,8}$. The $n^{-8 / 3}$ ROM scaling is shown for comparison. In this geometry the high-density plasma acts as a high-pass frequency filter and permits the generation of an attosecond pulse train without the need for further spectral filtering. This is shown in Fig. 2b, which confirms the temporal coherence of the CSE mechanism. It is worth noting that simulations have also shown that the characteristic spectral shape of CSE can also lead to attosecond pulse production even in the absence of such filtering ${ }^{7,8}$.

To investigate the generation of CSE in transmission from ultrathin foils we performed experiments using the Trident Laser Facility $^{23}$ at the Los Alamos National Laboratory. Nanometre-scale thickness diamond-like carbon (DLC) foil targets were placed in the laser focus normal to the incident beam (Fig. 1a), resulting in peak interaction intensities of $\sim 4 \times 10^{20} \mathrm{~W} \mathrm{~cm}^{-2}$. See Methods for laser pulse contrast considerations.

Figure 3 shows typical harmonic spectra obtained in transmission from $200 \mathrm{~nm}$ DLC targets using a Hitachi flatfield-gratingbased XUV spectrometer and a backthinned Andor CCD (chargecoupled device) detector. The spectrum emitted in a cone along the laser axis is characterized by a very slow decay to high orders (Fig. 3a). The off-axis spectrum at larger angles around the laser axis exhibits a more rapid decay to high orders (Fig. 3b). These two spectra combined demonstrate XUV-emission cone narrowing with increasing harmonic order ${ }^{24}$. The spectrum also exhibits a low-frequency cutoff $25<n_{\text {co }}<26$. The presence of this lowfrequency cutoff is a clear indication of the maintenance of a near-solid-density plasma at the time of CSE generation for $200 \mathrm{~nm}$ foils and confirms CSE from the front surface of the foil. This is in excellent agreement with hydrodynamic and PIC simulations for our exact pulse profile and interaction intensity. Spectra from 


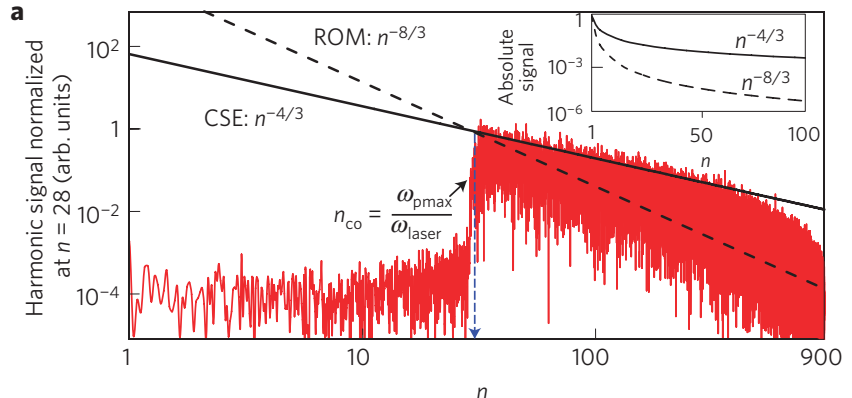

b

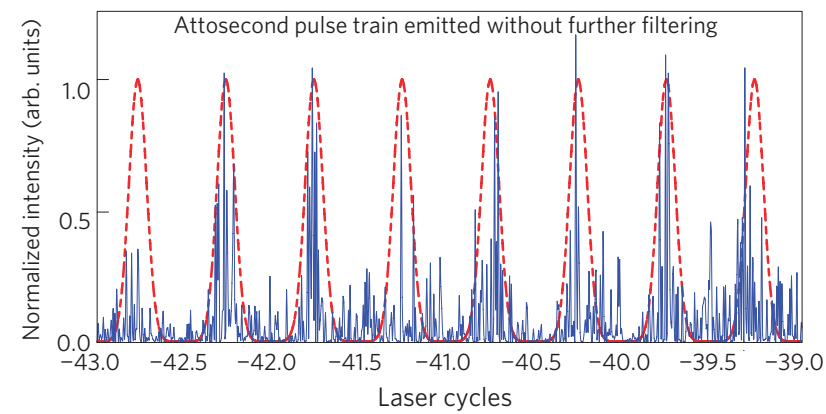

Figure 2 | PIC code simulations for CSE in transmission from normal incidence interactions. a,b, Spectrum (a) and corresponding pulse train (b) from the 1D simulation ${ }^{20}$ generated during the temporal interval -200 to $-100 \mathrm{fs}$ (cycles -60 to -30 ) on the rising edge of a $500 \mathrm{fs} / 150$ cycle pulse centred on $0 \mathrm{fs}$ (that is, -75 cycles (early) to +75 cycles (late)), with $a_{0}=20$, in normal incidence on a $200 \mathrm{~nm}$ foil at maximum density $N_{\max }=800 N_{c}$. See the Methods for the definition of $a_{0}$ and $N_{c}$. To verify the scaling of the emission, the data have been normalized at

$n_{\text {co }}=\omega_{\text {pmax }} / \omega_{\text {Laser }}=800^{1 / 2}=28$, corresponding to a low-frequency cutoff harmonic order (where $\omega_{\text {Laser }}$ is the laser frequency). The solid and dashed black lines correspond to $I(n)_{\mathrm{CSE}} \propto n^{-4 / 3}$ and $I(n)_{\mathrm{ROM}} \propto n^{-8 / 3}$ scalings, as labelled. The inset shows the relative absolute scaling for the CSE and ROM mechanics. For harmonic orders $<n_{\text {co }}$ emission is strongly suppressed owing to the opacity of the solid density plasma, which here corresponds to the maximum target density with $n_{\text {co }}=28$. It is important to note that the $n^{-4 / 3}$ scaling shown here is a fit to the simulation. We have chosen this temporal window of the driving pulse as it most clearly shows the CSE mechanism. It is important to note that the observed spectral scaling from the simulation is due to a combination of both the CSE mechanism and the evolution of the spectrum over the entire interaction. The dashed red line in b is a guide to see the attosecond pulse structure. See Supplementary Information for a more general discussion on CSE and the dynamic evolution of the emitted spectral shape for $500 \mathrm{fs}$ pulses and $50 \mathrm{fs}$ pulses using both 1D and 2D simulations.

$125 \mathrm{~nm}$ foils were also studied and exhibit no low-frequency cutoff in this spectral range, indicating a significantly reduced interaction density due to the lower target thickness.

Figure 4 shows harmonic spectra corrected for system transmission (normalized to $n=33$ ) from normal-incidence interactions for $200 \mathrm{~nm}$ (red triangles) and $125 \mathrm{~nm}$ (open blue diamonds) DLC foils. The relative efficiency scaling for $n<70$ is significantly shallower than the $n^{-8 / 3}$ expected for the ROM mechanism ${ }^{4,5}$ in both cases, and is consistent with CSE being the dominant emission mechanism. A very rapid rollover for $n>70$ is observed, again consistent with the CSE mechanism. The scaling of the absolute signal with foil thickness is shown in the inset of Fig. 4. No harmonic signal was observed for $50 \mathrm{~nm}$ DLC targets. The harmonic signal level increases by an average factor of $5.1 \pm 0.3$ for an increase in target thickness from 125 to $200 \mathrm{~nm}$. This marked signal increase is in good agreement with predictions for CSE signal variation with density scale length ${ }^{7,8}$.
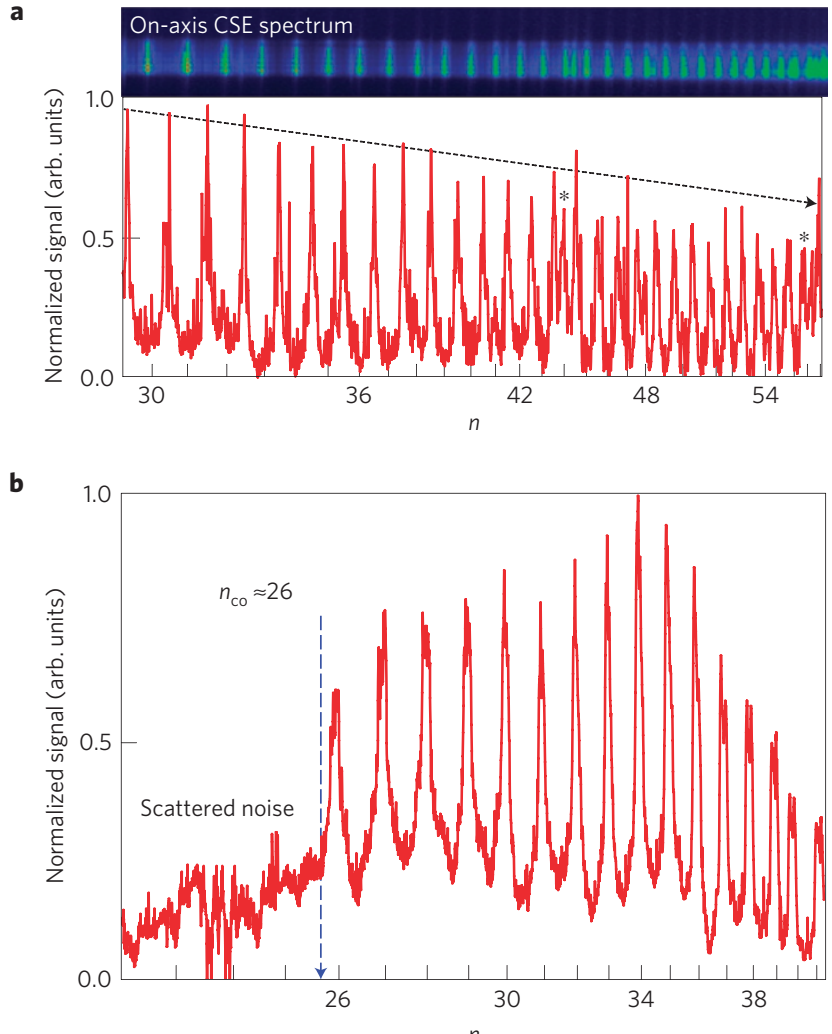

Figure 3 | Harmonic spectra in transmission from 200-nm DLC foils. a, A typical CCD image and spectrum obtained in transmission along the laser propagation axis from $200 \mathrm{~nm}$ foils ( 0 mrad to $52 \pm 8 \mathrm{mrad}$ half-cone angle, spectral lines marked with asterisks) collected using an adjustable gold focusing optic. $\mathbf{b}$, The spectrum obtained off the laser propagation axis ( $35 \pm 5$ to $140 \pm 5$ mrad halo). Strong suppression of lower-order harmonics $(n<26)$ is observed, implying a maximum target density

$N_{\max }>25^{2} N_{c}=625 N_{c}$ at the time of generation. See Supplementary Information for experimental set-ups.

The on-axis cone angle of $\sim 1 \mathrm{keV}$ emission $(n=1,000$, see Methods) is observed to be $10 \pm 1 \mathrm{mrad}$ at full-width at halfmaximum. A key feature of this profile is the presence of a lower intensity wide-angle $120 \pm 10 \mathrm{rad}$ scattered halo. This feature is also observed in two-dimensional (2D) simulations ${ }^{21}$ and is due to the reduction in the coherence of the generation mechanism during the later stages of the $500 \mathrm{fs}$ pulse. See Supplementary Information for a description of this measurement and discussion on how foil evolution affects the absolute conversion efficiencies observed in this experiment.

A tantalizing prospect for a bright attosecond source ${ }^{25,26}$ such as the one presented here is the potential for increasing focused intensities significantly beyond those of the driving laser. Previous work on the ROM mechanism has shown that substantial increases in peak intensity can be obtained owing to the spatio-temporal compression of the incident electromagnetic radiation through the frequency upshifting process ${ }^{27}$. The significantly slower decay of CSE spectra $\left(n^{-4 / 3}\right)$ when compared with those produced by the ROM mechanism $\left(n^{-8 / 3}\right)$ indicates the potential for substantial increases in peak intensity, well beyond those predicted for ROM scaling under idealized conditions ${ }^{8}$.

Strongly beamed XUV/X-ray harmonic emission with scaling consistent with the CSE mechanism is observed in transmission from ultrathin solid foils. The ability to generate CSE extending to the X-ray regime in transmission is shown to be made possible by the unique electron dynamics driven by the intense laser field 


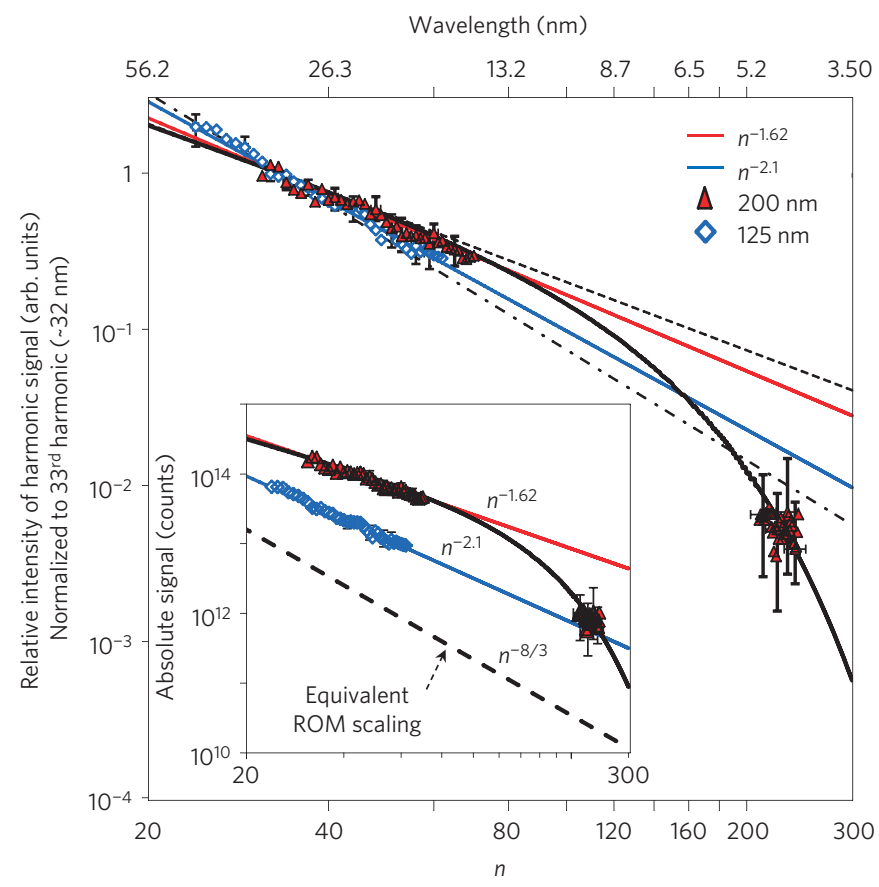

Figure 4 | Harmonic spectra from DLC foils normalized at $\boldsymbol{n}=\mathbf{3 3}$. Harmonics from $200 \mathrm{~nm}$ (red triangles) and $125 \mathrm{~nm}$ (open blue diamonds) foils are spatially and spectrally integrated and corrected for system

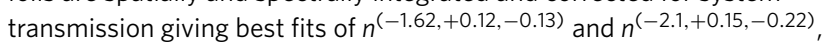
respectively, for $n<70$. The gap in the spectrum is due to spectrometer transmission. The bars represent uncertainty due to shot-to-shot fluctuations and propagation of errors from spectral deconvolution. The dashed $\left(n^{-(1.49)}\right)$ and dot-dashed $\left(n^{-(2.25)}\right)$ lines correspond to maxima (for $200 \mathrm{~nm}$ ) and minima (for $125 \mathrm{~nm}$ ) fits to the uncertainty for $n<70$, respectively, and essentially represent the extrema of the total uncertainty for this experiment. The curved solid black line serves as a guide to the spectral shape of the emission from the $200 \mathrm{~nm}$ foils. The inset shows the absolute count levels for $125 \mathrm{~nm}$ and $200 \mathrm{~nm}$ DLC targets and compares these with the relative signal strength expected from a corresponding ROM $\left(n^{-8 / 3}\right)$ source (that is, for equivalent signal level at $\left.n=1\right)$. The observed absolute energy in a single harmonic can be estimated as $200 \pm 50 \mu \mathrm{J}$ at $20 \mathrm{~nm}$ (58th harmonic) for $200 \mathrm{~nm}$ DLC foils and can be considered a lower bound due to the limited detection aperture in our experiment. Further studies of the spatio-temporal evolution of the interaction will be required to optimize conversion efficiency. Simulations show that the scattered halo observed in the kiloelectronvolt beam is a direct result of using a 500 fs pulse. See Supplementary Information for a detailed discussion on this topic.

at the front surface of the relativistic plasma. These dynamics allow for dense nanobunches (that is, $10^{9} \mathrm{~m}$ scale) to be formed and accelerated on ultrafast timescales $\left(\ll 10^{15} \mathrm{~s}\right)$. The observation of harmonic spectra in transmission from ultrathin foils with characteristic CSE scaling is clear evidence that the unique dynamics provided during relativistic laser plasma interactions can produce and rapidly accelerate dense attosecond electron bunches suitable for the emission of bright, coherent XUV/X-ray radiation in a convenient, practical geometry (see Methods).

\section{Methods}

Laser-pulse contrast. The Trident Laser Facility ${ }^{23}$ at Los Alamos National Laboratory is a Nd:glass $\left(\lambda_{\mathrm{L}}=1,053 \mathrm{~nm}\right)$ system delivering laser pulses with an energy of $E_{\mathrm{L}}=80 \mathrm{~J}$ in $500 \mathrm{fs}$ focused to a near-diffraction-limited focal spot using an $F / 3$ off-axis parabolic mirror. With this system a laser peak to pre-pulse level

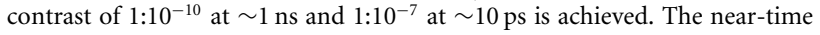
(sub $10 \mathrm{ps)}$ ) shot-to-shot variation in pulse contrast was measured using a single-shot third-order autocorrelator, showing typical values of $\sim 1: 10^{-4}$ at 2 ps.
It should be noted that contrast-enhancing plasma mirrors typical of relativistic laser-plasma-based harmonic generation were not required.

Simulations. To study the CSE generation mechanism in normal incidence in transmission we have performed a parametric study using two simulations (1D, 3-P PICWIG (ref. 20) and 2D PSC (ref. 21) PIC codes) to identify the exact generation mechanism and anticipated spectral shape of the emission in transmission. In the manuscript, $a_{0}$ is the normalized vector potential, where $a_{0}=\sqrt{I_{\mathrm{L}} \lambda_{\mathrm{L}}^{2} /\left(1.37 \times 10^{18} \mathrm{~W} \mu \mathrm{m}^{2} \mathrm{~cm}^{-2}\right)}$, and $I_{\mathrm{L}}$ and $\lambda_{\mathrm{L}}$ are the focused intensity and wavelength of the driving laser. The critical density for the incident driving laser, $N_{\mathrm{c}}$, is defined as $N_{\mathrm{c}}=\left(\epsilon_{0} m_{e} \omega_{\mathrm{L}} / e^{2}\right) c$ and $\omega_{\mathrm{L}}$ is the laser frequency, which itself depends on the electron charge $e$ and mass $m_{e}$.

Kiloelectronvolt beam footprint. The CSE $>1 \mathrm{keV}$ was examined using an image plate detector and edge filter stack. The beam profile is determined by background subtraction and correction for multiple scans of the image plate. The image plate was placed behind a pair of magnets with an average $B$-field of $0.44 \mathrm{~T}$ to deflect charged particles and was filtered using $50 \mu \mathrm{m} \mathrm{Al}$ foil. To confirm the presence of kiloelectronvolt radiation a spectral step filter was used over the lower half of the image plate $(100 \mu \mathrm{m} \mathrm{CH})$. See Supplementary Information for the experimental set-up and further details.

Received 27 January 2012; accepted 3 September 2012; published online 7 October 2012

\section{References}

1. Bulanov, S. V., Naumova, N. M. \& Pegoraro, F. Interaction of an ultrashort, relativistically strong laser pulse with an overdense plasma. Phys. Plasmas 1, 745-757 (1994).

2. Lichters, R., Meyer-ter-Vehn, J. \& Pukhov, A. Short-pulse laser harmonics from oscillating plasma surfaces driven at relativistic intensity. Phys. Plasmas 3, 3425-3437 (1996).

3. Gibbon, P. Harmonic generation by femtosecond laser-solid interaction: A coherent water-window light source? Phys. Rev. Lett. 76, 50-53 (1996).

4. Baeva, T., Gordienko, S. \& Pukhov, A. Theory of high-order harmonic generation in relativistic laser interaction with overdense plasma. Phys. Rev. E 74, 046404 (2006).

5. Dromey, B. et al. Harmonic generation in the relativistic limit. Nature Phys. 2, 456-459 (2006).

6. Zepf, M. et al. High harmonics from relativistically oscillating plasma surfaces - a high brightness attosecond source at keV photon energies. Plasma Phys. Contr. F. 49, B149-B162 (2007).

7. An der Brügge, D. \& Pukhov, A. Enhanced relativistic harmonics by electron nanobunching. Phys. Plasmas 17, 033110 (2010).

8. Pukhov, A., An der Brügge, D. \& Kostyukov, I. Relativistic laser plasmas for electron acceleration and short wavelength radiation generation. Plasma Phys. Control. Fusion 52, 124039 (2010).

9. Nomura, Y. et al. Attosecond phase locking of harmonics emitted from laser-produced plasmas. Nature Phys. 5, 124-128 (2009).

10. Dromey, B. et al. Diffraction limited performance and focusing of high harmonics from relativistic plasmas. Nature Phys. 5, 146-152 (2006).

11. Dromey, B. et al. Bright multi-keV harmonic generation from relativistically oscillating plasma surfaces. Phys. Rev. Lett. 99, 085001 (2007).

12. Quéré, F. et al. Coherent wake emission of high-order harmonics from overdense plasmas. Phys. Rev. Lett. 96, 125004 (2006).

13. Naumova, N. M., Nees, J. A., Sokolov, I. V, Hou, B. \& Mourou, G. A. Relativistic generation of isolated attosecond pulses in a $\lambda^{3}$ focal volume. Phys. Rev. Lett. 93, 195003 (2004).

14. An der Brügge, D. \& Pukhov, A. Theory of attosecond pulses from relativistic surface. Preprint at http://arxiv.org/abs/1111.4133v1 (2011).

15. Zepf, M. et al. Role of the plasma scale length in the harmonic generation from solid targets. Phys. Rev. E 58, R5253-R5256 (1998).

16. Tarasevitch, A., Lobov, K., Wunsche, C. \& von der Linde, D. Transition to the relativistic regime in high order harmonic generation. Phys. Rev. Lett. 98, 103902 (2007).

17. Thaury, C. \& Quéré, F. High-order harmonic and attosecond pulse generation on plasma mirrors: Basic mechanisms. J. Phys. B 43, 213001 (2010).

18. George, H., Quéré, F., Thaury, C., Bonnaud, G. \& Martin, Ph. Mechanisms of forward laser harmonic emission from thin overdense plasmas. New J. Phys. 11, 113028 (2009).

19. Teubner, U. \& Gibbon, P. High-order harmonics from laser-irradiated plasma surfaces. Rev. Mod. Phys. 81, 445-479 (2009).

20. Rykovanov, S. G., Geissler, M., Meyer-ter-Vehn, J. \& Tsakiris, G. D. Intense single attosecond pulses from surface harmonics using the polarization gating technique. New J. Phys. 10, 025025 (2008).

21. Ruhl, H. in Introduction to Computational Methods in Many-Body Physics (eds Bonitz, M. \& Semkat, D.) (Rinton, 2006).

22. Popov, K. I. et al. Vacuum electron acceleration by tightly focused laser pulses with nanoscale targets. Phys. Plasmas 16, 053106 (2006). 
23. Batha, S. et al. TRIDENT high-energy-density facility experimental capabilities and diagnostics. Rev. Sci. Instrum. 79, 10F305 (2008).

24. An der Brügge, D. \& Pukhov, A. Propagation of relativistic surface harmonics radiation in free space. Phys. Plasmas 14, 093104 (2007).

25. Krausz, F. \& Ivanov, M. Attosecond physics. Rev. Mod. Phys. 81, 163-234 (2009).

26. Mourou, G.A. \& Tajima, T. More intense shorter pulse. Science 331, 41-42 (2011).

27. Gordienko, S., Pukhov, A., Shorokhov, O. \& Baeva, T. Coherent focusing of high harmonics: A new way towards the extreme intensities. Phys. Rev. Lett. 94, 103903 (2005)

\section{Acknowledgements}

The authors would like to acknowledge and thank the Trident laser staff for continued support throughout multiple experimental campaigns. B.D. acknowledges support from the EPSRC through grants and a Career Acceleration Fellowship.

\section{Author contributions}

B.D., S.R., C.L.S.L. and M.Z. prepared the initial manuscript. All authors contributed comments and revision to the final version. S.R. provided simulation and analytical theory. The multiple experiments discussed in this manuscript were performed and/or analysed by all other authors equally. This research strand was led by B.D. and R.H. under the larger projects of B.M.H and J.C.F.

\section{Additional information}

Supplementary information is available in the online version of the paper. Reprints and permissions information is available online at www.nature.com/reprints. Correspondence and requests for materials should be addressed to B.D.

\section{Competing financial interests}

The authors declare no competing financial interests. 